\title{
MRI brain tumor segmentation: A forthright image processing approach
}

\author{
Noor Elaiza Abd Khalid', Muhammad Firdaus Ismail ${ }^{2}$, Muhammad Azri AB Manaf ${ }^{3}$, \\ Ahmad Firdaus Ahmad Fadzil ${ }^{4}$, Shafaf Ibrahim ${ }^{5}$ \\ ${ }^{1}$ Faculty of Computer and Mathematical Sciences, UiTM Shah Alam, Malaysia \\ ${ }^{2,3}$ Faculty of Health Science, UiTM Selangor, Malay sia \\ ${ }^{4,5}$ Faculty of Computer and Mathematical Sciences, UiTM Melaka, Malay sia
}

\begin{tabular}{l}
\hline Article Info \\
\hline Article history: \\
Received Oct 19, 2019 \\
Revised Dec 29, 2019 \\
Accepted Feb 25, 2020 \\
\hline
\end{tabular}

\section{Keywords:}

Confusion matrix

MRI

Segmentation

Tumor

\begin{abstract}
Brain tumor is a collection of cells that grow in an abnormal and uncontrollable way. It may affect the regular function of the brain since it grows inside the skull region. As a brain tumor can be possibly led to cancer, early detection in computed tomography (CT) or magnetic resonance imaging (MRI) scanned images are crucial. Thus, this paper proposed a forthright image processing approach towards detection and localization of brain tumor region The approach consists of a few stages such as pre-processing, edge detection and segmentation. The pre-processing stage converts the original image into a greyscale image, and noise removal if necessary. Next, the image is enhanced using image enhancement techniques. It is then followed by edge detection using Sobel and Canny algorithms. Finally, the segmentation is applied to highlight the tumor with morphological operations towards the affected region in the MRI images. The in-depth analysis is measured using a confusion matrix. From the results, it signifies that the proposed approach is capable to provide decent segmentation of brain tumor from various MRI brain images.
\end{abstract}

This is an open access article under the $\underline{C C B Y-S A}$ license.

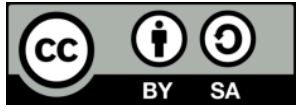

\section{Corresponding Author:}

Noor Elaiza Abd Khalid, Faculty of Computer and Mathematical Sciences, UiTM Shah Alam, Malaysia.

Email: elaiza@tmsk.uitm.edu.my

\section{INTRODUCTION}

The presence of uncontrolled and irregular cells' growth within the skull is referred to a brain tumor. Brain cancer is a severe brain disease. Cancer arises when the body cells start to divide uncontrollably, and spread surrounds the other components [1]. The tumor can cause severe pain as it can either directly or indirectly damage the brain cells when the tumor grows and swells [2]. Generally, high-risk disease such as brain tumor can be detected and diagnosed by various imaging processing techniques which are available to be applied on several imaging modalities such as computed tomography (CT) scan [3], medical resonance imaging (MRI) [4], brain biopsy and others. The availability of these medical tools helps for tumor detection which will enhance some features of the tumors such as size, border, shape, texture, and classification [5].

MRI images are often processed via computing tools such as MATLAB [6] using various algorithms to define and segment the brain tumor in an image [7]. While modern techniques such as machine learning [8] have shown tremendous success towards accurately segmenting different features in medical images, classic image processing techniques are vastly employed in previous research and subsequently should not be completely negated. Different research has employed image processing methodology to identify and provide decent segmentation of the brain tumor in MRI images. For example, previous research 
proposes a methodology that consists of multiple stages of image processing methodology [9, 10]. The stages encompass image acquisition, pre-processing, applying morphological operation, and finally segmenting the desired feature by employing different segmentation techniques such as clu stering and thresholding [11]. Another study by [12] emphasizes the employment of several types of edges detection algorithms such as Robert, Prewitt, and Sobel. These algorithms can be utilized to define edges within MRI which can be later used to allow easier segmentation towards an image.

This paper, therefore, proposed a forthright MRI brain tumor segmentation that utilizes straightforward image processing methodology such as pre-processing, edge detection algorithm, and morphological operation. This paper highlights the utilization of straightforward image pre-processing techniques such as image enhancement and edge detection techniques to enhance the features of the brain tumor. Segmentation via thresholding and morphological operation are also employed to finally segment the tumor region in MRI images. The results of the segmentation approach are evaluated by employing the confusion matrix using the manually annotated brain tumor as the ground truth.

\section{RESEARCH METHOD}

The proposed approach comprises three stages of pre-processing, edge detection, and segmentation as illustrated in Figure 1. The pre-processing of the given MRI image is initially performed, followed by the edge detection of the brain, and finally, the segmentation which shows the tumor area vividly [3]. As an alternative method of segmentation, the K-means clustering algorithm was introduced. The algorithm may also reveal the other essential tis sues and edges, in addition to the tumor area.

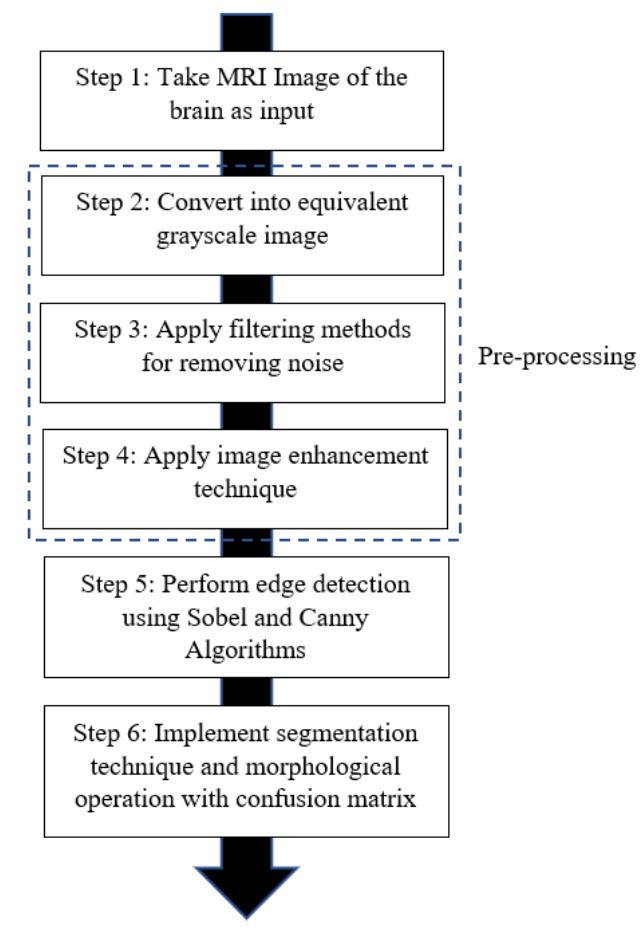

Figure 1. Proposed brain tumor segmentation algorithm

\subsection{Pre-processing}

This stage is aimed at eliminating noise and enhancing the image's clarity to suit the purpose. The pre-processing functions are defined as follows:

\subsubsection{RGB to grayscale conversion}

This conversion indicates that the image may consist of the shades of grey [13]. All the colors such as red, green, blue elements have a similar intensity towards the RGB space and it same goes to the gray color. The grayscale image contains the values of the grayscale but some MRI images are primary RGB material [5]. Thus, these images need to be converted into greyscale image where the pixel values are 
ranging from 0 to 255 pixel values. The ' 0 ' range describes the pure black color and the '255' range defines a pure white shade.

\subsubsection{Noise removal using median filtering}

Eliminating the noise presented in the image is known as the filtering technique. Some noise may creep into the image during the image conversion from RGB to gray. Thus, filtering is applied to removes the noise. It is also used to eliminate the noise such as salt and pepper from the converted images [6]. The value of the center pixel is compared with the average of the intensity values in the adjacent pixels [7].

\subsubsection{Image enhancement}

The obtained image may have poor contrast which affects the images. Contrast enhancement seems to be important when the images come with poor contrast. In this case, the grey level of each pixel is scaled to improving the contrast. Thus, the MRI images visualization is improved through the contrast enhancement technique [5].

\subsection{Edge detection techniques}

The main purpose of the edge detection techniques can be defined to determine the boundaries of the desired object within the image in accessing image processing. The edge detection algorithms are performed by judging the sudden increase or decrease of each pixel intensity and visualizing only those sudden alterations in the pixels. This difference of the pixel will be passed through an adequate convolution mask and the result will be described the edges of the image $[14,15]$. There are two types of edge detection techniques will be described below such Sobel and Canny edge detection method.

\subsubsection{Sobel edge detection technique}

Sobel operator can also be known as gradient operator. The relative gradient magnitude can be achieved by implementing this operator in every point of the input image. Generally, there are two setting convolution mask c=2 of Sobel operator which is $\mathrm{Mx}$ and $\mathrm{My}$ as it achieved through the size of $3 \times 3$ convolution masks as shown in Figure 2. The term of convolution can be defined as the process of appending individual image element to its local kernel-weighted neighbor.

\begin{tabular}{|l|l|l|}
\hline-1 & 0 & 1 \\
\hline-2 & 0 & 2 \\
\hline-1 & 0 & 1 \\
\hline
\end{tabular}

$\mathrm{M}_{\mathrm{x}}$

\begin{tabular}{|l|l|l|}
\hline-1 & -2 & -1 \\
\hline 0 & 0 & 0 \\
\hline 1 & 2 & 1 \\
\hline
\end{tabular}

$\mathrm{M}_{\mathrm{y}}$

Figure 2. Sobel operator: Vertical and horizontal masks

\subsubsection{Canny edge detection technique}

Canny edge detection is one of the most powerful and frequently used image processing tools to detect the edges [8]. Generally, the main approach of the Canny edge detection method associated with the smoothing of an input image such as noise removal. Besides, the purpose of this method step involved transforming the blurred or irregular edges of an image into sharp edges to achieve regular and continuous edges. The process of this principle takes place by preserving all the local maxima that present in the gradient image and removing unnecessary things.

The important part of the edge detection method is the removal of Gaussian noise from the input image by applying the Gaussian filter model. Therefore, the essential implementing of the Gaussian filter will be explained in detail below. Firstly, the Gaussian filter is applied to sharpen the image as well as to decrease the noise at the edge detectors by brightening the image [16]. Then, every pixel of the input image will be passed through a size of 5x5 Gaussian masks as given in (2). As the all edge detection outcomes are affected by noise, thus it is essential to remove the noise as to avoid the false detection led by presence of noise. The Gaussian filter kernel of size $(2 k+1) \times(2 k+1)$ is shown in (1).

$$
\begin{gathered}
H_{i j}=\left(2 \pi \sigma^{2}\right)^{-1} \exp \left(-(i-(k+1))^{2}+(j-(k+1))^{2} / 2 \sigma\right) ; \\
1 \leq i, j \leq(2 k+1)
\end{gathered}
$$


The Gaussian filter size used to filter the image is $5 \times 5$ Gaussian masks. It is used as to build the adjacent image as the value pixel of $\mathrm{A}$ is presently being processed and value $\mathrm{B}$ is achieved after it undergoes the Gaussian mask filter. It is important for every pixel of the image must undergo Gaussian mask such given in (2).

$$
B=\frac{1}{159}\left[\begin{array}{rrccc}
2 & 4 & 5 & 4 & 2 \\
4 & 9 & 12 & 9 & 4 \\
5 & 12 & 15 & 12 & 5 \\
4 & 9 & 12 & 9 & 4 \\
2 & 4 & 5 & 4 & 2
\end{array}\right] * A
$$

Moreover, it is essential to note that the selection of the Gaussian kernel size will affect the detector's performance. The bigger the mask's size, the smaller sensitivity of the detector in recognizing the noise. Besides, the image will become more blurred as the Gaussian filter kernel size is greater. The choice of Gaussian mask $5 \times 5$ is preferable good commonly for most cases in image filtering. However, it may differ relied on certain situations [17].

\subsection{Segmentation}

The most essential task and step in image processing is image segmentation [18]. It is the used to extract the desired details from an image. Medical image segmentation automation has set up various applications in different regions such as patient evaluation, scheduling of case management and operation assisted by computers. Three main techniques that widely used in image segmentation are region-based approach, thresholding (boundary) approach and edge-based approach [19]. In this project, the global thresholding approach has been used for image segmentation.

Generally, the thresholding techniques described the pixels are assigned to from depending on the range values in which image pixel lies. Besides, this method is preferable to apply as the method is easier and more popular as compared with other methods approach segmentation. The component of thresholding techniques describes the $\mathrm{g}(\mathrm{x}, \mathrm{y})$ that represent the location of every individual pixel of the image and $\mathrm{T}$ as the threshold value. The value of threshold $\mathrm{T}$ is defined in this algorithm as the value of ongoing pixel $f(x . y) \leq T$ then the pixel $g(x . y)$ is assigned as 0 . In contrast, the pixel $g(x . y)$ is assigned as 1 . The segmentation image can be achieved when all the values of "g" are presented $[15,20]$. The pixels are partitioned when it comes to determine its intensity values.

Commonly, the thresholding method is performed using the global thresholding method. In this method, only one threshold values are implemented on all images. This technique is taking place when the defected portion of the pixel value and the background value are dependable over the whole image. The value of global thresholding is applied to an appropriate threshold value such in (3) and (4).

$$
\begin{aligned}
& T: g(x, y)=1, \text { if } f(x, y)>T \\
& T: g(x, y)=0, \text { if } f(x, y) \leq T
\end{aligned}
$$

\section{RESULTS AND DISCUSSION}

The dataset employed are retrieved from multimodal brain tumor segmentation (BraTS) 2017 [21, 22]. A dataset which consists of 150 different slices of brain MRI image using different modalities such as T1, T1-CE, T2, and FLAIR are examined to select the correct slices of the dataset that contain the brain abnormality (tumor) anomaly.

\subsection{Segmentation results}

To begin the processing of an input image, the application of the graphical user interface (GUI) in the MATLAB is running. Since the MRI images are not in grayscale, all the images are converted into grayscale images. MRI images mostly succumb to noise due to operator performance, equipment, and environment [23]. To remove the noise, the mean filter [24] was applied. Here the example of contrast enhancement. It has been shown in Figure 3.

Edge detection is used to separate the well-defined regions of MRI images. Sobel and Canny algorithm are implemented for this purpose. The edges of the brain are clearly presented in each algorithm. The Sobel algorithm detects only the brain image boundaries while the Canny algorithm detects the entire region in the brain. Outputs for both algorithms are demonstrated in Figure 4 and Figure 5 respectively. 

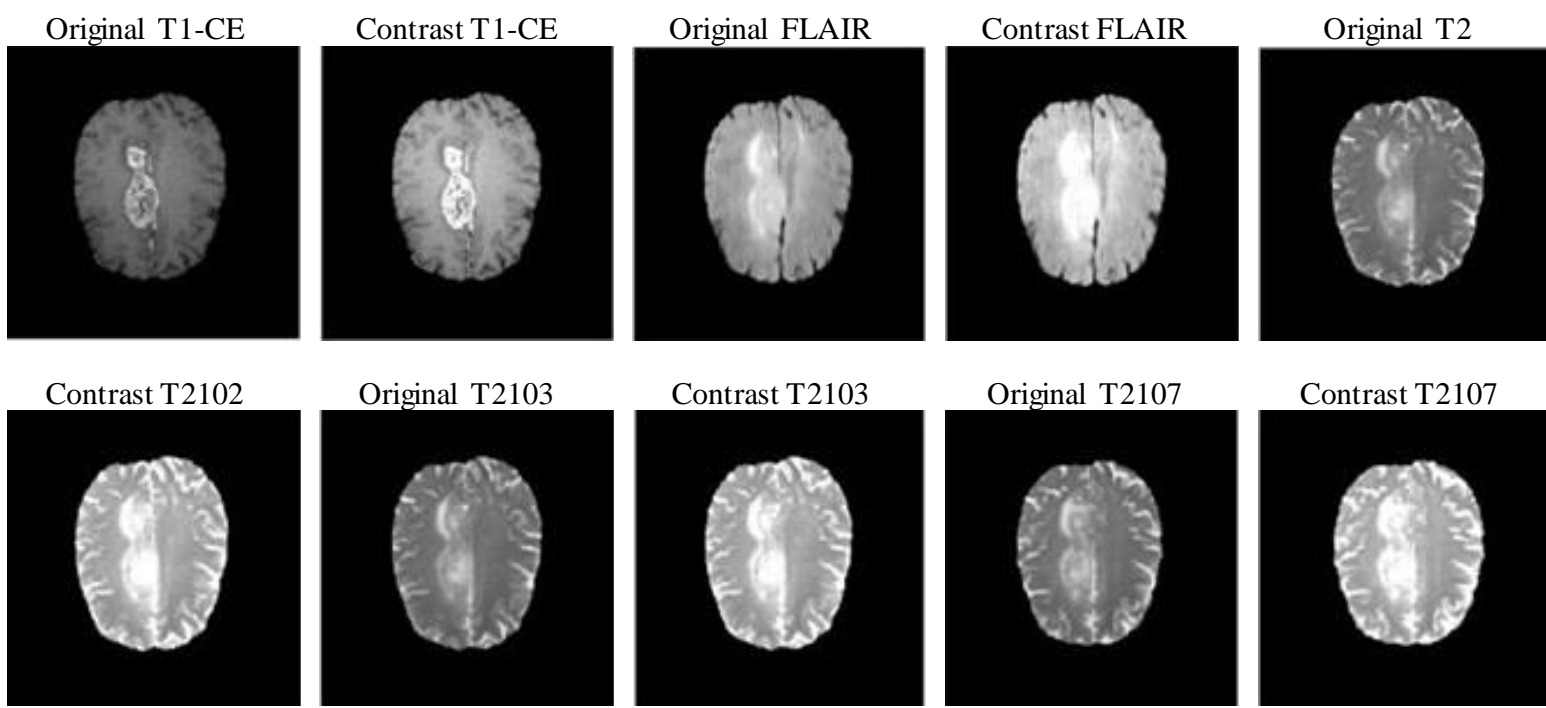

Contrast T2107

Figure 3. Contrast enhanced MRI image
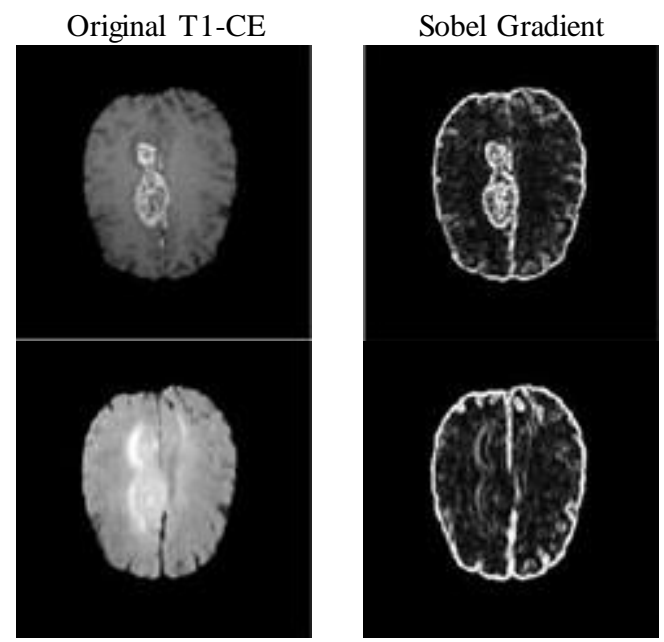

Edge detected

Figure 4. Sobel operation edge detection MRI image
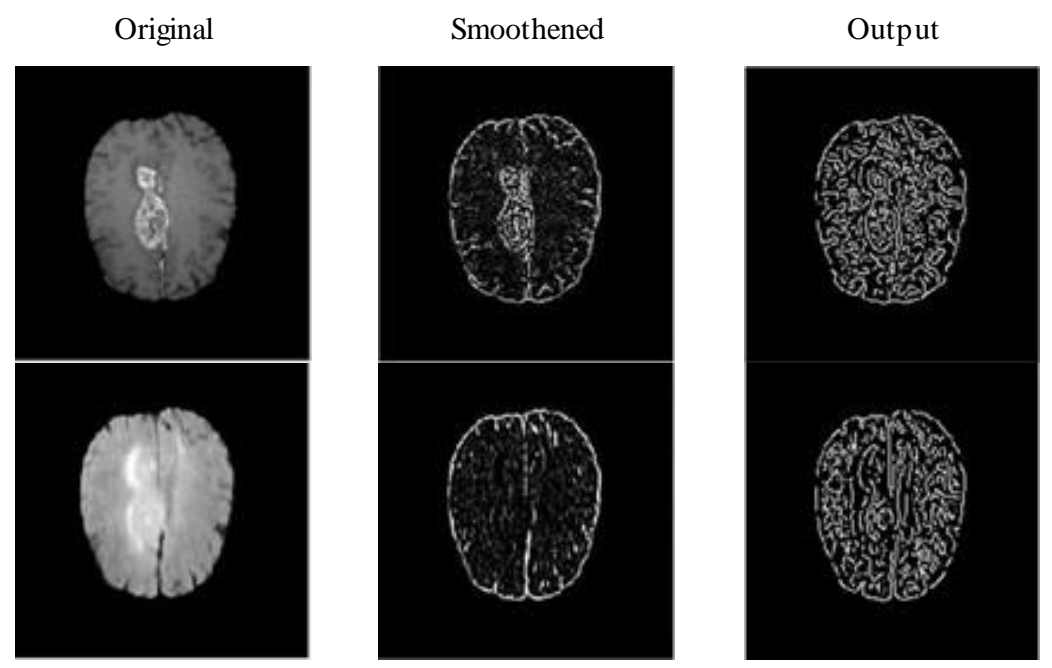

Output with Canny detector

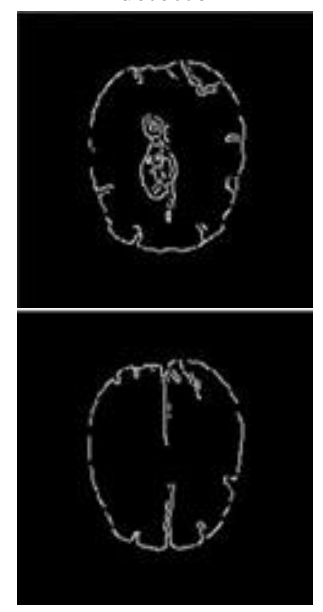

Figure 5. Canny operation edge detection MRI image 
Finally, the segmentation process is carried out by employing global thresholding. The results of the segmentation are demonstrated in Table 1. The final output image predicts only the tumor regions present in the MRI brain image. The region that presented with the tumor exhibit the white pixels forming and it is displayed in Table 2.

Table 1. Segmentation results

\begin{tabular}{cccc}
\hline \multicolumn{2}{c}{ Threshold segmentation for T1-CE } & \multicolumn{2}{c}{ Threshold segmentation FLAIR } \\
\hline Original image & Threshold image & Original image & Threshold image \\
\hline & & & \\
\hline
\end{tabular}

Table 2. MRI brain tumor segmentation final output

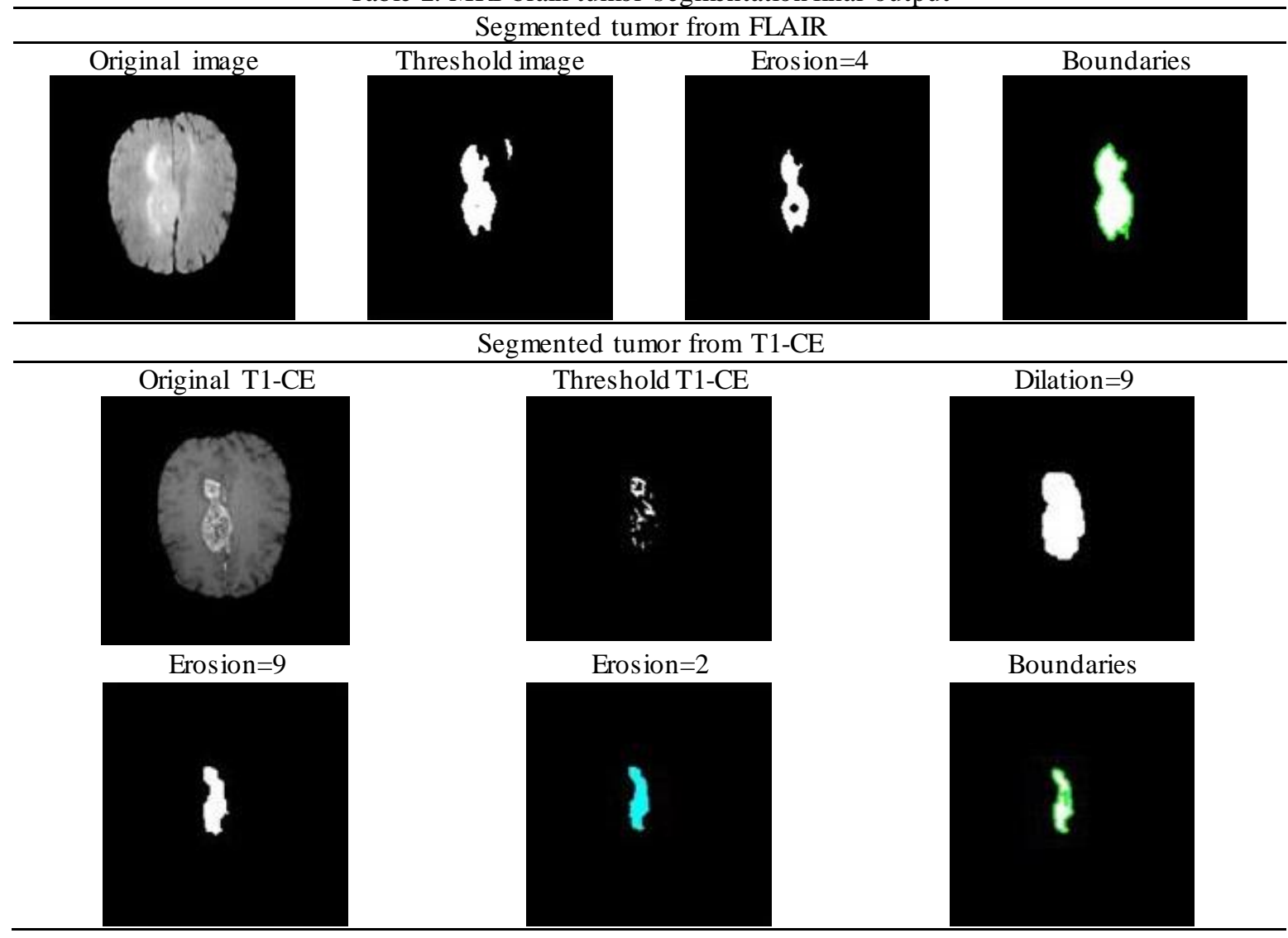

\subsection{Confusion matrix}

The chosen slices of the MRI image are then processed to segment the desired feature. The results are deliberated in terms of sensitivity or true positive rate (TPR), specificity or true negative rate (TNR), accuracy (ACC), precision or positive predictive value (PPV), and negative predictive value (NPV) [25]. Table 3 illustrates the confusion matrix from the segmentation results.

From the Table 3 below, segmentation results from Flair and T1-CE modality show decent values in terms of accuracy (ACC) compare to T2 slices. It can be suggested that brain tumor segmentation can be accomplished by using a set of forthright image processing method when employing Flair and T1-CE modalities. However, the limitation of this method can is apparent when employing T2 slices MRI brain 
images. Both slices' tumor segmentation albeit is high in terms of positive predictive value (PPV), unable to turn in a decent value in terms of accuracy.

Table 3. Confusion matrix

\begin{tabular}{lccccc}
\hline & TPR & TNR & ACC & PPV & NPV \\
\hline Flair & 0.14 & 1.0 & 0.76 & 1.0 & 0.75 \\
T1-CE & 0.04 & 1.0 & 0.74 & 1.0 & 0.73 \\
T2 (Slice 102) & 0.0 & 0.03 & 0.03 & 0.0 & 1.0 \\
T2 (Slice 107) & 0.0 & 0.03 & 0.03 & 0.0 & 1.0 \\
\hline
\end{tabular}

\section{CONCLUSION}

MRI images are common tools for brain tumor detection. In this paper, a forthright image processing technique is proposed and demonstrated to segment brain tumor from MRI brain images. From the result congregated, it can be suggested that while recent image processing advancement such as employment of machine Learning and Artificial Intelligence are more preferred, the techniques and processes from straightforward image processing can still produce decent brain tumor segmentation results. This forthright image processing approach can also be further protracted with other approaches such as research on the textures of different MRI modalities.

\section{ACKNOWLEDGEMENTS}

The research was supported by Ministry of Education Malaysia (MoE), and Universiti Teknologi MARA through the Fundamental Research Grant Scheme (FRGS) (600-IRMI/FRGS 5/3 (215/2019)).

\section{REFERENCES}

[1] N. K. E. Abbadi and Z. F. Shoman, "Detection and recognition of brain tumor based on DWT, PCA and ANN," Indonesian Journal of Electrical Engineering and Computer Science, vol. 18, no. 1, pp. 56-63, 2020.

[2] A. Sehgal, S. Goel, P. Mangipudi, A. Mehra, and D. Tyagi, "Automatic brain tumor segmentation and extraction in MR images," IEEE Conference on Advances in Signal Processing (CASP), pp. 104-107, 2016.

[3] A. Mustaqeem, A. Javed, and T. Fatima, "An efficient brain tumor detection algorithm using watershed \& thresholding based segmentation," Int. J. Image, Graph. Signal Process., vol. 4, no. 10, pp. 34-39, 2012.

[4] S. Ibrahim, N. E. A Khalid, and M. Manaf, "CAPSOCA: Hybrid technique for nosologic segmentation of primary brain tumors," Indonesian Journal of Electrical Engineering and Computer Science, vol. 16, no. 1, pp. 267-274, 2019.

[5] D. Cavouras, P. Prassopoulos, and N. Pantelidis, "Image analysis methods for solitary pulmonary nodule characterization by computed tomography," Eur. J. Radiol., vol. 14, no. 3, pp. 169-172, 1992.

[6] D. D. Kumar, K. S. Priy a, S. Vandhana, and S. J. Subashini, "Brain tumour image segmentation using MATLAB," vol. 1, no. 12, pp. 447-451, 2015.

[7] A. Sivaramakrishnan and M. Karnan, "A novel based approach for extraction of brain tumor in MRI images using soft computing techniques," International Journal of Advanced Research in Computer and Communicating Engineering, vol. 2, no. 4, pp. 2-5, 2013

[8] S. Ibrahim, M. H. C. Rozan, and N. Sabri, "Comparative analysis of support vector machine (SVM) and convolutional neural network (CNN) for white blood cells' classification," International Journal of Advanced Trends in Computer Science and Engineering, vol. 8, no. 1.3 SI, pp. 394-399, 2019.

[9] J. Kong et al., "A novel approach for segmentation of MRI brain images," IEEE Mediterranean Electrotechnical Conference, pp. 525-528, 2006.

[10] E. E. M. Azhari, M. M. M. Hatta, Z. Z. Htike, and S. L. Win, "Brain tumor detection and localization in magnetic resonance imaging," Int. J. Inf. Technol. Converg. Serv., vol. 4, no. 1, pp. 2231-1939, 2014.

[11] J. Kaur, S. Agrawal, and R. Vig, "A comparative analysis of thresholding and edge detection segmentation techniques,” Int. J. Comput. Appl., vol. 39, no. 15, pp. 29-34, 2012.

[12] R. Rulaningty as and K. Ain, "Edge detection for brain tumor pattern recognition," IEEE Int. Conf. Instrumentation, Commun. Inf. Technology Biomed. Eng., pp. 23-25, 2009.

[13] S. Ibrahim, N. Wahab, A. F. A. Fadzil, N. A. A. Mangshor, and Z. Ahmad, "Automatic classification of paddy leaf disease," Indonesian Journal of Electrical Engineering and Computer Science, vol. 16, no. 2, pp. 767-774, 2019.

[14] N. Nandhagopal and S. Ganesh, "Automatic detection of brain tumor through magnetic resonance image," International Journal of Advanced Research in Computer and Communication Engineering, vol. 2, no. 4, pp. 1647-1651, 2013.

[15] V. Y. Borole, S. S. Nimbhore, and S. S. Kawthekar, "Image processing techniques for brain tumor detection: A review," Int. J. Emerg. Trends Technol. Comput. Sci., vol. 4, no. 2, pp. 28-32, 2015.

[16] M. Sudharson, S. R. T. Rajapandiyan, and P. U. Ilavarasi, "Brain tumor detection by image processing using MATLAB," Middle-East J. Sci. Res., vol. 24, pp. 143-148, 2016. 
[17] A. Al-Badarneh, H. Najadat, and A. M. Alraziqi, "A classifier to detect tumor disease in MRI brain images," Proc. IEEE/ACM Int. Conf. Adv. Soc. Networks Anal. Mining, pp. 784-787, 2012.

[18] S. Ibrahim, N. A. Zulkifli, N. Sabri, A. A. Shari, and M. R. M. Noordin, "Rice grain classification using multi-class support vector machine (SVM)," International Journal of Artificial Intelligence, vol. 8, no. 3, pp. 215-220, 2019.

[19] R. S. Kapse, S. S. Salankar, and M. Babar, "Literature survey on detection of brain tumor from MRI images," IOSR J. Electron. Commun. Eng., vol. 10, no. 1, pp. 80-86, 2015.

[20] A. Anand and H. Kaur, "Survey on segmentation of brain tumor: A review of literature," International Journal of Advanced Research in Computer and Communication Engineering, vol. 5, no. 1, pp. 79-82, 2016.

[21] B. H. Menze et al., "The multimodal brain tumor image segmentation benchmark (BRATS)," IEEE Transactions on Medical Imaging, vol. 34, no. 10, pp. 1993-2024, 2015.

[22] S. Bakas et al., "Advancing the cancer genome atlas glioma MRI collections with expert segmentation labels and radiomic features," Nature Scientific Data, vol. 4, pp. 1-13, 2017.

[23] S. Vaishali, K. K. Rao, and G. S. Rao, "A review on noise reduction methods for brain MRI images," International Conference on Signal Processing and Communication Engineering Systems, pp. 363-365, 2015.

[24] A. Hazra, A. Dey, S. K. Gupta and Md. Abid Ansari, "Brain tumor detection based on segmentation using," International Conference on Energy, Communication, Data Analytics and Soft Computing, pp. 425-430, 2017.

[25] A. Jose and D. S. Juliet, "Recent advances and investigation of efficient computer aided diagnosis systems for CT images in liver cancer detection," International Journal of Advanced Trends in Computer Science and Engineering, vol. 8, no. 3, pp. 343-348, 2019. 